\title{
ARTICLE OPEN Yeast longevity promoted by reversing aging-associated decline in heavy isotope content
}

\author{
Xiyan $\mathrm{Li}^{1}$ and Michael P Snyder ${ }^{1}$
}

Dysregulation of metabolism develops with organismal aging. Both genetic and environmental manipulations promote longevity by effectively diverting various metabolic processes against aging. How these processes converge on the metabolome is not clear. Here we report that the heavy isotopic forms of common elements, a universal feature of metabolites, decline in yeast cells undergoing chronological aging. Supplementation of deuterium, a heavy hydrogen isotope, through heavy water $\left(\mathrm{D}_{2} \mathrm{O}\right)$ uptake extends yeast chronological lifespan (CLS) by up to $85 \%$ with minimal effects on growth. The CLS extension by $\mathrm{D}_{2} \mathrm{O}$ bypasses several known genetic regulators, but is abrogated by calorie restriction and mitochondrial deficiency. Heavy water substantially suppresses endogenous generation of reactive oxygen species (ROS) and slows the pace of metabolic consumption and disposal. Protection from aging by heavy isotopes might result from kinetic modulation of biochemical reactions. Altogether, our findings reveal a novel perspective of aging and new means for promoting longevity.

npj Aging and Mechanisms of Disease (2016) 2, 16004; doi:10.1038/npjamd.2016.4; published online 18 February 2016

\section{INTRODUCTION}

Normal aging is accompanied by progressive metabolism deterioration. ${ }^{1}$ Understanding the basic mechanisms involved in aging and how the process can be manipulated will provide useful insights and treatment for this universal process. Currently, adjusting and diverting metabolic flows serve as a major objective to delay aging. Genetic manipulation of a range of metabolic regulators are implicated in lifespan extension in experimental organisms. ${ }^{1}$ In budding yeast $S$. cerevisiae, these include suppression of anabolism through mTOR inhibition, ${ }^{2}$ activation of catabolism through $\mathrm{AMPK}^{3}$ and sirtuins activation, ${ }^{4}$ and enhancement of bioenergetics through mitochondria. ${ }^{5}$ In parallel, calorie restriction ( $C R$, or dietary restriction, $D R)$, as a typical environmental intervention, works equally well as, or in certain cases, even better than genetic manipulation to extend lifespan. ${ }^{6}$ Modulation of metabolism through nutrient uptake may thus represent a deserving avenue of anti-aging intervention.

Two discrete types of aging could be recognized in the budding yeast: ${ }^{7}(1)$ replicative aging which is marked by a gradual loss of dividing capability in single cells; and (2) chronological aging which is defined by diminished survival of nondividing cells. Both aging processes may be retarded by limiting nutrients, including carbon sources (through $C R)^{4,8}$ and amino acids (AAs; through $\mathrm{DR})^{2,6}$ pinpointing the importance of nutrient availability and metabolism in regulating lifespan. Examining the metabolome of yeast cells may provide direct clues to underlying mechanisms involved in aging.

\section{RESULTS}

The yeast metabolomic decline during aging

To reveal age-associated changes of metabolome, we first examined the metabolome from yeast cells. For this study, we focused primarily on chronological aging which can be analyzed by standard assays. ${ }^{9}$ In this assay, yeast cells are grown to stationary phase, incubated for varying degrees of time, and the percentage of viable cells determined. We examined yeast cells undergoing chronological aging at 3 time points, using an untargeted liquid chromatography-coupled mass spectrometry (LC-MS)-based metabolomics approach (Figure 1a-d). At day 7, the survival ratio drops to around $50 \%$ of the level at day 3 for both DBY746 and W303, ${ }^{9}$ two strains commonly used in chronological aging studies. A total of 2,936 high-confidence metabolites exhibited distinctive clustering with respect to each time point for both strains $(R 2 Y>0.98, Q 2>0.95)$, indicating a clear trend in age-associated metabolome changes (Figure $1 a, b$ ). The contribution of individual metabolites to the multivariate regression clustering model (O2PLS-DA) was correlated with its distance to the placeholder for each time point group (Figure 1c,d). Of 237 metabolites with a KEGG entry, 58 metabolites that showed a decreasing trend with time were shared in both strains, and over-represents a few metabolic pathways, including arginine and proline metabolism (urea cycle) and oxidative phosphorylation (Supplementary Table S2), two processes for energy generation and waste disposal in catabolism that also decline in aging. ${ }^{10,11}$ In contrast, 17 metabolites with an increasing trend were found in both strains and showed no enrichment for any metabolic pathways (Supplementary Table S2). These observed changes in the metabolome over chronological aging corroborates with the decline in general metabolism in other experimental organisms. ${ }^{1}$

In particular, the intracellular levels of 16 free AAs started to decline as late as day 5, reflecting a natural depletion of nutrients in aging population (Figure 1c,d), which also agrees with previous observations that branched-chain AAs decline with replicative aging. ${ }^{12,13}$ In both strains, glutamine $(G \ln )$ showed the fastest depletion, as judged by its closest proximity to the day 3 placeholder (Figure 1d and Supplementary Figure S1a).

\footnotetext{
${ }^{1}$ Department of Genetics, Stanford University School of Medicine, Stanford, CA, USA. Correspondence: MP Snyder (mpsnyder@stanford.edu)

Received 1 December 2015; accepted 10 December 2015
} 
The metabolite isotope content decline during aging

Aside from measuring the levels of metabolites, we also examined the isotopic content of metabolites which has not been analyzed previously. Heavy stable isotopes represent a natural small portion of common elements found in biological systems, but may markedlly affect the kinetics of biochemical reactions. ${ }^{14}$ When operated with high mass resolution (resolving power at 100,000 in this study), mass spectrometry is able to distinguish the subtle mass differences between molecular forms that contain different isotopes for the same element, including most common forms such as ${ }^{13} \mathrm{C},{ }^{15} \mathrm{~N}$ and ${ }^{2} \mathrm{H}$ (or D; see Supplementary Figure S2b for an example). Current technical capacity of mass spectrometry can only achieve sufficient separation of isotopic species for a given metabolite with a molecular mass below $300 \mathrm{Da}$ and with an a

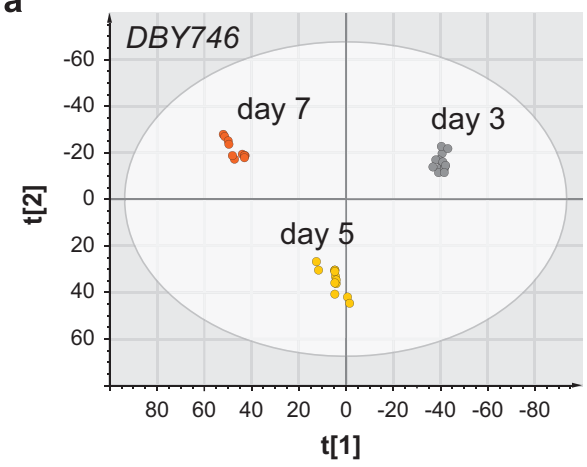

C
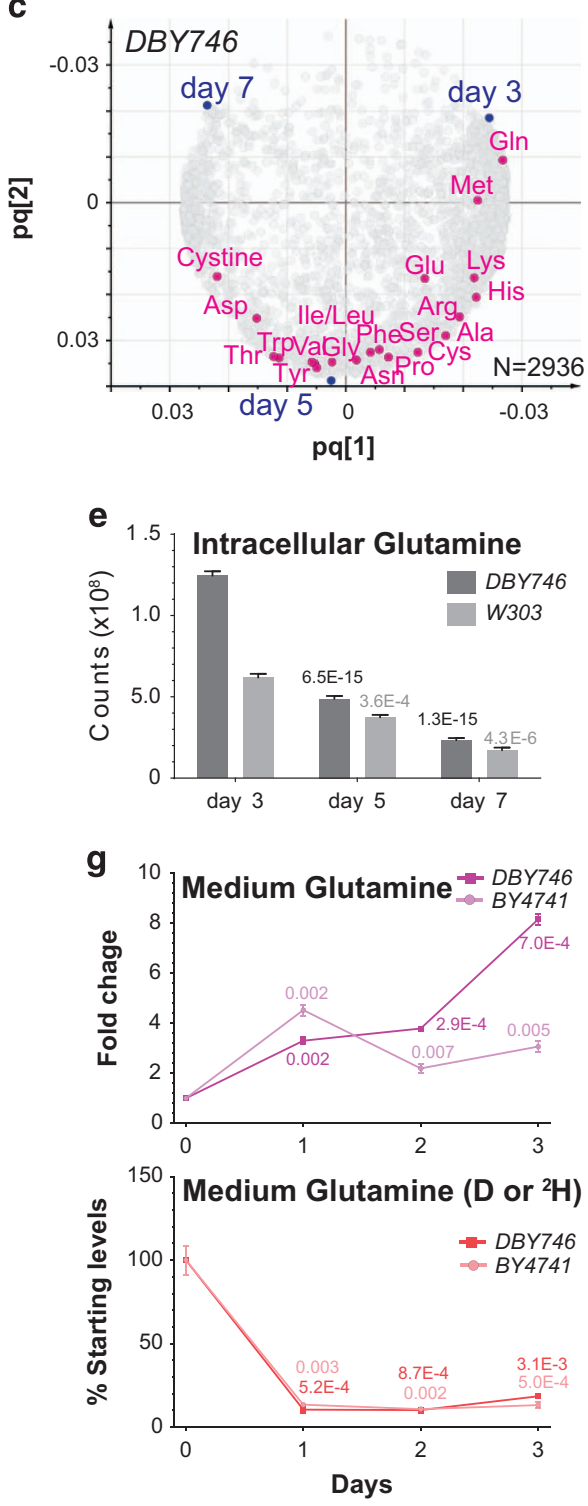

b

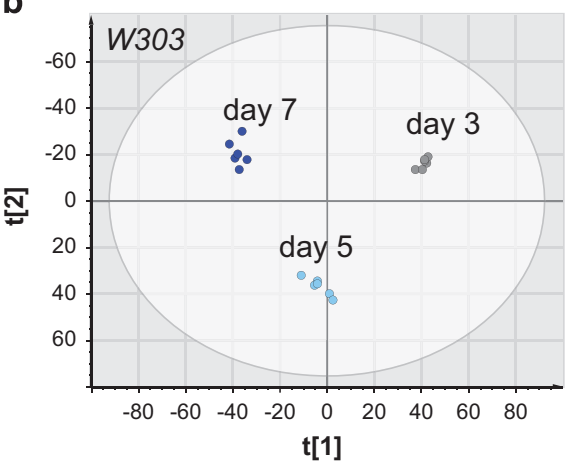

d
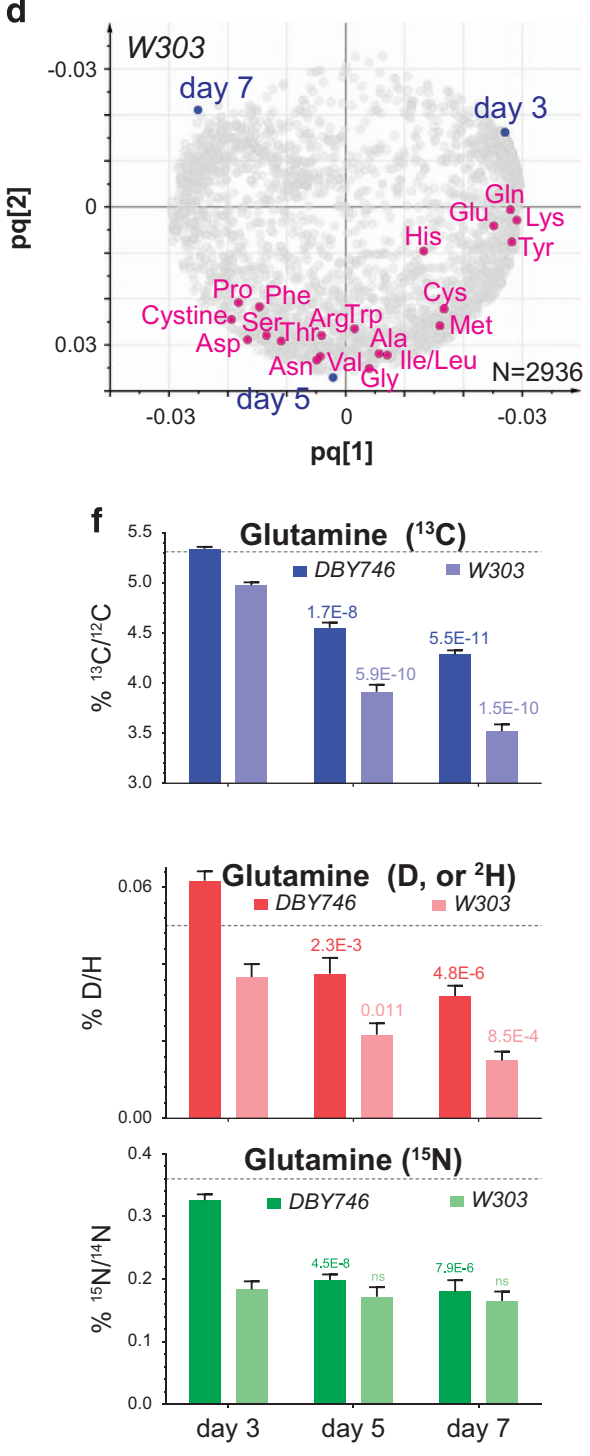
Figure 1. Aging-associated decline in heavy isotopic metabolites in yeast revealed by LC-MS-based metabolomics. See also Supplementary Figure S1, Supplementary Table S2. (a, b), The intracellular metabolome from yeast cells was examined by LC-MS when cells have reached stationary phase at day 3,5 , and 7 after inoculation ( $n=6$ for each time point). The O2PLS-DA clustering for two strains (DBY746, W303) is shown for DBY746 in a $(R 2 Y=0.983, Q 2=0.979)$, and for $\mathrm{W} 303$ in $\mathbf{b}(\mathrm{R} 2 \mathrm{Y}=0.985, \mathrm{Q} 2=0.955)$. $X$ and $Y$ axis are reversed for clarity of comparison. (c, d) Contribution of individual metabolites to the clustering models in $\mathbf{a}, \mathbf{b}$, respectively. Each circle represents a metabolite (mass feature). All twenty proteinogenic amino acids and cysteine are highlighted in magenta. Our method does not distinguish isoleucine and leucine. (e) The plot of the intracellular levels of glutamine (Gln) in $\mathbf{c}$, $\mathbf{d}$. The $P$ values of two-tailed unequal variance $t$-test are shown above each bar for comparison with levels at day 3 for each strain. Error bars =s.e.m. The same type $t$-test was applied in all other analyses in this manuscript unless otherwise noted. (f) The relative monoisotopic abundances of glutamine that contains one ${ }^{13} \mathrm{C},{ }^{2} \mathrm{H}(\mathrm{D})$ or ${ }^{15} \mathrm{~N}$, respectively, normalized to the most abundant mass species composed of all light isotopes $(n=6)$. Values were manually calculated in Thermo XCalibur. The $t$-test $P$ values are shown above each bar for comparison with levels at day 3 for each strain. Dotted lines indicate the relative monoisotopic abundances of medium glutamine. Error bars = s.e.m. NS, not significant. (g) The levels of glutamine and its D-isotopic form in the culture medium in the first 3 days after inoculation for two strains (DBY746, BY4741, $n=3$ each). The $t$-test $P$ values are shown above each bar for comparison with starting medium levels. Error bars $=$ s.e.m.

abundance that expands over three orders of magnitude in MS counts. Consequently, we only manually measured the heavy isotope-containing forms of small metabolites, and report the results for 20 AA because they represent an intracellular metabolite group that is essential for protein synthesis and subject to stringent surveillance at the metabolic level. ${ }^{15}$

Strikingly, the relative abundance of heavy isotope-containing metabolites exhibited an aging-associated trend as well. Whereas less abundant AAs showed an increase in heavy isotope content, more abundant AAs declined in heavy isotope content, suggesting an aging-associated decline in the metabolome (Supplementary Figure S1c). The most prominent AA showing a declining trend was glutamine. Both ${ }^{13} \mathrm{C}$ and ${ }^{2} \mathrm{H}$-containing forms of glutamine declined to $50 \%$ at day 7 from day 3 as a portion of total glutamine in both strains (Figure 1f). In contrast, glutamate (Glu), a closely related AA, exhibited no obvious trends in relative abundance of all three isotopes despite similar decline in total Glu in the same period of time (Supplementary Figure S1d,e). The aging-associated Gln decline is unlikely due to the lack of availability, as the Gln levels actually increased by 3 to 8 -fold in the medium at day 3, as determined by LC-MS (Figure 1g). Conversely, medium Glu levels were rapidly depleted at the same time (Supplementary Figure S1f). The accumulation of Gln in medium likely results from deregulated metabolic waste disposal such as detoxification of ammonia. ${ }^{16}$ Given the prevalence and central importance of Gln in microbial metabolism, ${ }^{16,17}$ the agingassociated decline in the total and isotopic Gln may be a consequence of changes in multiple metabolic processes, including energy generation (e.g., oxidative phosphorylation) and waste disposal (e.g., catabolic amino-acid metabolism). We note that due to technical limitation and vast dynamic ranges of intracellular metabolite content during aging, not all AAs were suitable for this type of thorough measurement, as denoted by the gray color in Supplementary Figure S1c. Nevertheless, this finding raised the novel hypothesis that yeast cells may gradually lose the ability to retain heavy metabolites in aging.

Yeast chronological lifespan extension by heavy water uptake

For many abundant metabolites heavy isotope contents decrease during aging. We therefore tested whether the addition of heavy isotopes might extend chronological lifespan in yeast. We introduced heavy isotope nutrients through medium uptake into haploid and diploid yeast, and determined the mean chronological lifespan by quantifying the time required for surviving colony forming units in a stationary phase culture to drop to $50 \%$ (Supplementary Figure S2a), as previously reported..$^{9}$ Feeding yeast with ${ }^{2} \mathrm{H}$ (deuterium) through glucose was able to extend the chronological lifespan (CLS) significantly, but not to substantial extent (Figure 2c). This is likely due to the fact that the metabolic incorporation of deuterium through glucose only represents a small portion of hydrogen flux metabolism, therefore it only marginally affects cell metabolism and thus cell aging. We also reasoned that ${ }^{13} \mathrm{C}$ incorporation through single nutrient uptake may also fail to effectively affect carbon flux metabolism for similar reasons, as supported by yeast growth experiments (not shown) and noted in parallel stable isotope labeling experiments. $^{18}$

We then tested whether deuterium supplementation through alternative means extends yeast CLS. Because of the limited availability of deuterated AAs and the observation that medium Gln accumulation accompanied intracellular Gln declines during aging (Figure $1 e, g$ ), we used heavy water $\left(D_{2} O\right)$ as the vector to introduce deuterium into the metabolites. Heavy water directly suppresses chemical reactions that involve water splitting or forming, which make up 39\% all biochemical reactions in budding yeast. ${ }^{19}$ We observed substantial CLS extension in different yeast strains incubated with increasing doses of $\mathrm{D}_{2} \mathrm{O}$. $\mathrm{D}_{2} \mathrm{O}$ extended the CLS in a dose-dependent manner in the two haploid and one diploid strains tested, and the maximal CLS extension, upon $50 \%$ $\mathrm{D}_{2} \mathrm{O}$ treatment, was $85 \%(P=0.0001)$ for DBY746 (haploid, a strain commonly used for CLS studies $\left.{ }^{9}\right), 50 \%(P=4.1 \mathrm{E}-8)$ and $59 \%$ $(P=2.5 \mathrm{E}-7)$ for BY4741 (haploid, a strain used for the Yeast Genome Deletion Project), and BY4743 (a diploid strain related to BY4741), respectively (Figure 2b, also see Supplementary Table S1). Importantly, CLS extension was also observed when $\mathrm{D}_{2} \mathrm{O}$ was administrated after the yeast cells reached stationary phase (Supplementary Figure S2f), demonstrating that $\mathrm{D}_{2} \mathrm{O}$ uptake promotes longevity in both proliferative and nonproliferative growth stages.

The CLS extension is unlikely due to any potential influence of $\mathrm{D}_{2} \mathrm{O}$ on cell growth, as the initial colony formation capacity or cell volumes at the beginning of the lifespan assays were not substantially altered by $\mathrm{D}_{2} \mathrm{O}$ (Supplementary Figure $\mathrm{S} 2 \mathrm{~b}, \mathrm{c}$ ). Besides, according to previous study, $\mathrm{D}_{2} \mathrm{O}$ only had negligible effect on oxygen consumption in the dosage range used in this study. ${ }^{20}$ Furthermore, growth curve assays showed $\mathrm{D}_{2} \mathrm{O}$ only slightly affected the culture at the log phases (Supplementary Figure S2d,e), which substantially precedes when all cultures reached saturation phase at day 3, the first time point for all CLS assays. The growth capability of yeast cells did not appear to be affected even after prolonged exposure (40 days) to $\mathrm{D}_{2} \mathrm{O}$ (Supplementary Figure S2g,h), suggesting that the observed lifespan extension is unlikely the consequence of a biased selection for spontaneous mutations promoting fitness, which could skew the assay.

\section{Bypassing of genetic regulators by $\mathrm{D}_{2} \mathrm{O}$ in CLS extension}

To position the heavy isotope effects in relation to genetic CLS regulation, we tested whether $\mathrm{D}_{2} \mathrm{O}$ extends yeast CLS in the absence of known aging regulators (BY4741 background). We determined the mean CLS in yeast strains with gene deletions in GPR1, SIR2 or TOR1, each of which has a reported role in affecting 

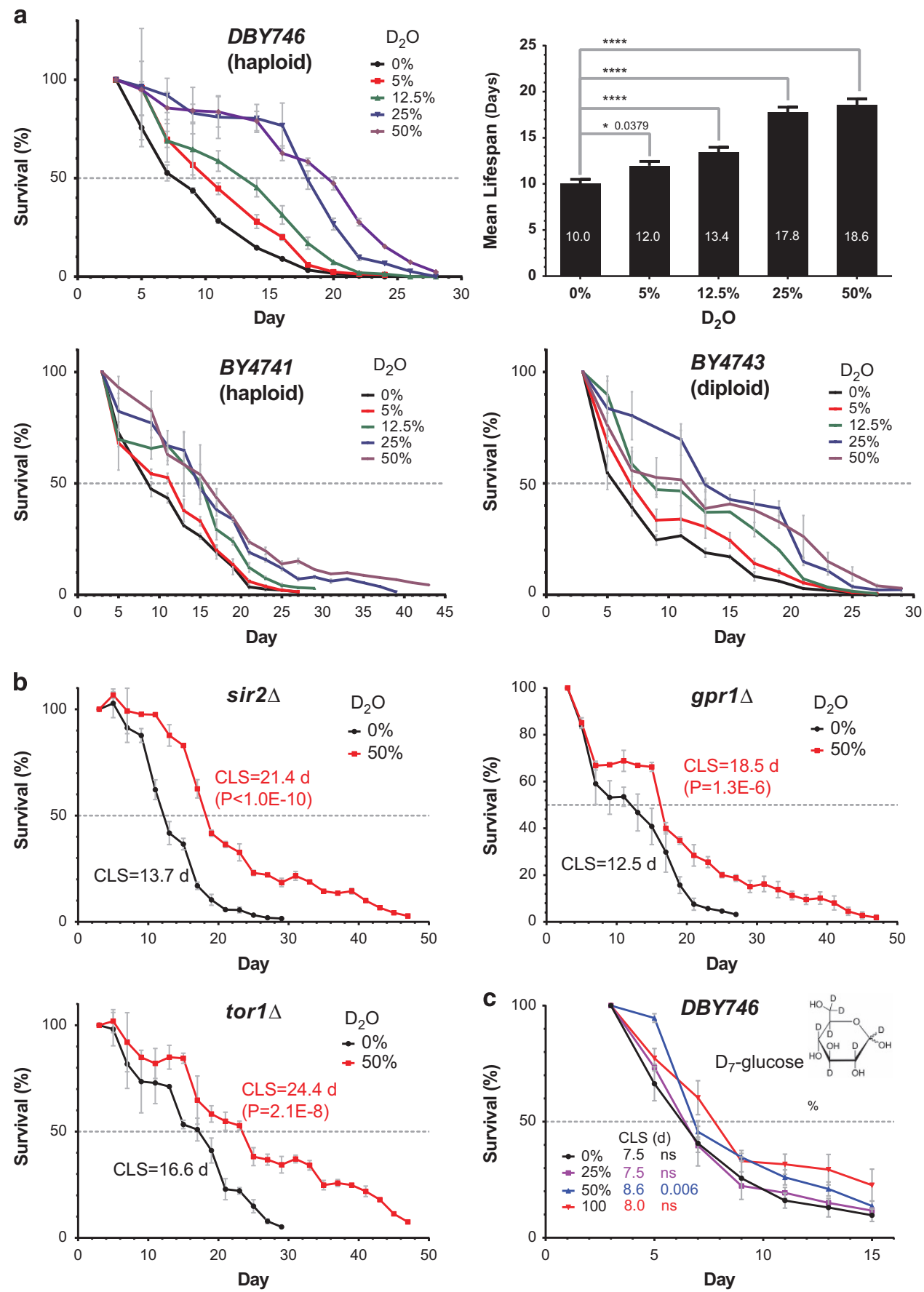

Figure 2. Chronological lifespan extension in yeast by metabolic introduction of heavy hydrogen isotopes deuterium through heavy water $\left(\mathrm{D}_{2} \mathrm{O}\right)$. See also Supplementary Figure S2. (a) Chronological survival curves for yeast (S. cerevisiae) strains DBY746, BY4741 and BY4743, respectively $(n=3)$. Chronological aging assays were performed in fortified SDC medium as described previously (see Materials and Methods) for several common laboratory strains. Fresh culture was used to inoculate SDC medium containing different amounts of $\mathrm{D}_{2} \mathrm{O}(\mathrm{v} / \mathrm{v})$. Colony formation was determined from day 3 and every 2 days thereafter until the survival rate dropped below $10 \%$ (representative images in Supplementary Figure S2A). At least three independent experiments were performed, and one is shown here. Top right panel shows mean lifespans (estimated by Kaplan-Meier survival analysis, shown inside each bar) of DBY746 strain in media containing $\mathrm{D}_{2} \mathrm{O}$. $P$ values (log-rank test after Bonferroni correction) of pair-wise comparisons are indicated above the bars $(*,<0.05$; $* * * *,<1 \mathrm{E}-4)$. Error bars $=$ s.e.m. $(\mathbf{b}) \mathrm{CLS}$ assays of three yeast mutants (BY4741 background) treated with $50 \% \mathrm{D}_{2} \mathrm{O}$, each with a deletion of a known aging regulator gene in yeast $(n=3)$. Two independent experiments were performed, and one is shown here. The mean lifespans and $P$ values are estimated by log-rank test and indicated on each panel. The experiments were from the same culture presented in a. Error bars =s.e.m. (c) Deuterium introduced through carbon sources (glucose) failed to extend CLS in yeast to a significant extent. The total glucose concentration is $2 \%$ (w/v) in all conditions. D7-glucose contains deuterium at all seven of nonexchangeable hydrogen positions (structure shown on right). The mean lifespans and $P$ values (log-rank test, versus $0 \% D_{7}$-glucose) are indicated with the same color of survival curves. NS, not significant. Error bars $=$ s.e.m. 
a
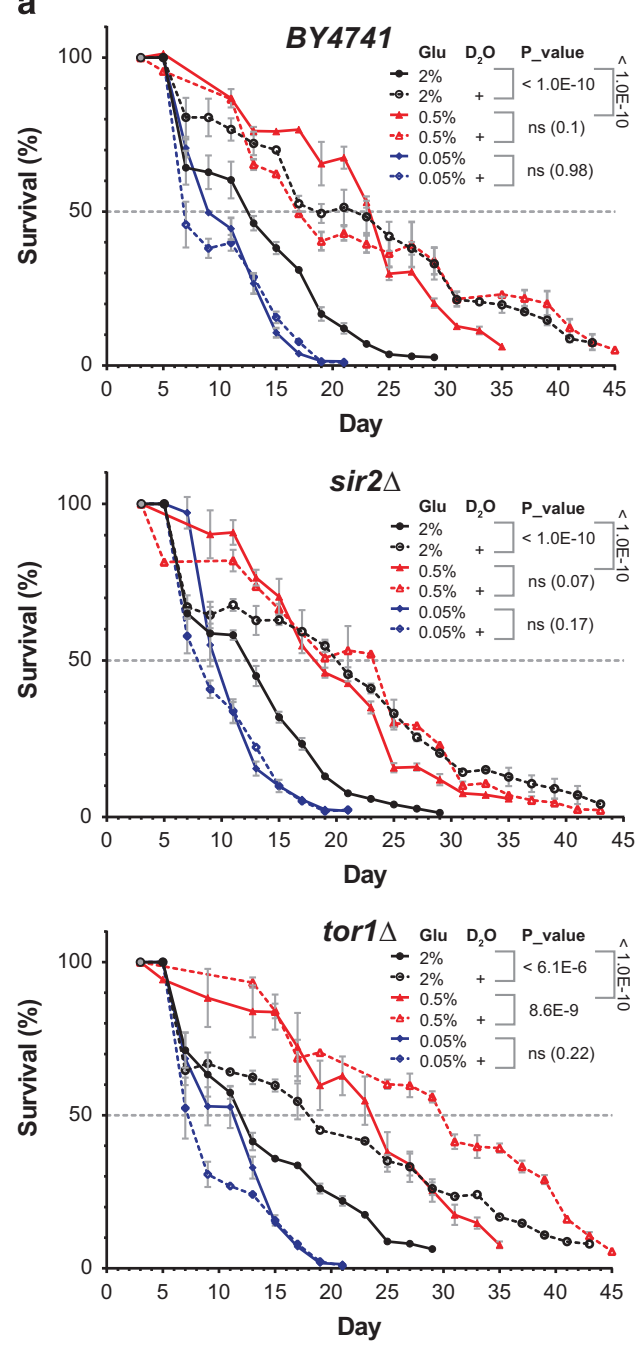

b Strain $\mathrm{D}_{2} \mathrm{O}$ CLS (d)

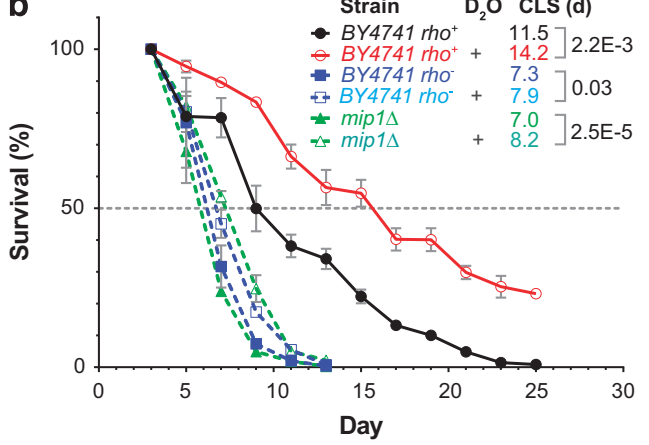

C

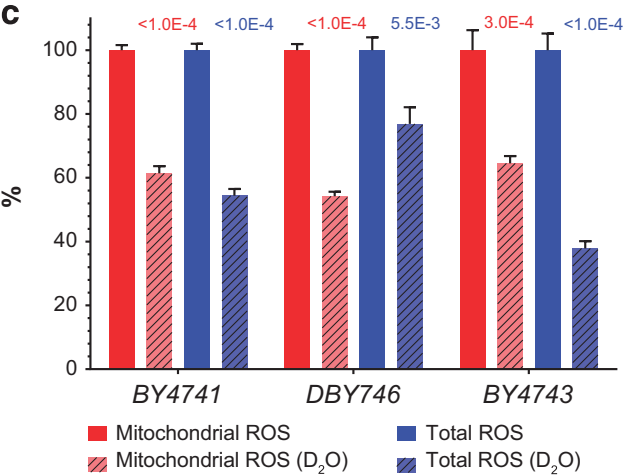

Figure 3. Heavy water extension of yeast chronological lifespan mediated by calorie restriction and mitochondria. See also Supplementary Figure S3. (a) CLS extension by $\mathrm{D}_{2} \mathrm{O}(50 \%)$ is abrogated by mild calorie restriction $(n=3)$. Two independent experiments were performed, and one is shown here. The $P$ values (log-rank test) for comparison with respective control $\left(\right.$ no $\left.D_{2} O\right)$ are indicated in the legends. The $P$ values (log-rank test) for comparison with $2 \%$ glucose are $<1.0 \mathrm{E}-5$ for all other five conditions. NS, not significant. Error bars $=$ s.e.m. (b) $\mathrm{CLS}$ extension by $\mathrm{D}_{2} \mathrm{O}(50 \%)$ is attenuated in yeast cells lacking mitochondria. Mitochondria-deficient petite strains (rho $\left.{ }^{-}\right)$were generated through EtBr treatment (see Materials and Methods). The deletion strain mip 1 $\triangle$ (BY4741 background) lacks the gene encoding the single subunit of the mitochondrial DNA polymerase in S. cerevisiae. Lifespans upon treatment with 50\% D2O is shown. The mean lifespans and $P$ values (log-rank test, versus BY4741 (rho $\left.{ }^{+}, 0 \%\right)$ are indicated with colors respective to legends. $n=3$. Error bars $=$ s.e.m. (c) Measurement of in vivo generation of reactive oxygen species (ROS). The ROS generation was continuously monitored by fluorescent dyes CellROX (total ROS) and MitoSOX (mitochondrial ROS) on a plate reader for three strains treated with $\mathrm{D}_{2} \mathrm{O}(50 \%)(n=3)$. The ROS generation, as calculated by area under curve calculation in Supplementary Figure S3, was normalized to no $\mathrm{D}_{2} \mathrm{O}$ controls for each strain (control as $100 \%$ ). The $P$ values (two-tailed $t$-test, unequal variance) are indicated on graph. Error bars = s.e.m.

yeast lifespan. $4,9,13,21,22$ Consistent with previous studies, we confirmed that strains with a deficiency in TOR1 are long-lived (44.9\%, $P$ value 3.9E - 8) (Figure $2 \mathrm{~b}$ ). The mean CLS of each mutant strain was extended by $\mathrm{D}_{2} \mathrm{O}$ by a level comparable to that in the wild-type strain (gpr1 $148.3 \%$; sir2 $\Delta, 56.5 \%$; tor $1 \Delta, 47.0 \%$, versus $50 \%$ in BY4741) (Figure 2b). These results showed that $\mathrm{D}_{2} \mathrm{O}$ bypasses these genes in its capability to extend CLS, and thus $\mathrm{D}_{2} \mathrm{O}$ likely acts on downstream or in parallel to these genetic regulations in promoting longevity, which also corroborates its minimal effects on yeast growth (Supplementary Figure S2b,e).

\section{CLS extension by $\mathrm{D}_{2} \mathrm{O}$ mediated by metabolism}

Heavy isotopes greatly affect the kinetics of biochemical reactions, the chemical basis for metabolism en masse. The metabolic decline in aging may lead to decline in heavy isotope content in the metabolome (Figure 1). Conversely, because $\mathrm{D}_{2} \mathrm{O}$ suppresses all hydrolysis reactions, which are mostly catabolic, $\mathrm{D}_{2} \mathrm{O}$ treatment may retard aging-related metabolic deterioration. To test whether CLS extension by $\mathrm{D}_{2} \mathrm{O}$ is mediated by metabolism, we applied $\mathrm{D}_{2} \mathrm{O}$ treatment under calorie restriction $(C R)$ conditions in yeast. $\mathrm{CR}$ works as an effective environmental intervention to activate catabolism and extend yeast lifespan. ${ }^{6}$ Different $C R$ regimens act through different intracellular pathways in yeast. ${ }^{4,23,24}$ Consistent with previous reports, we observed that mild CR (0.5\% glucose) extended the mean CLS in all three genetic conditions (BY4741 wild-type, sir $2 \Delta$ and tor $1 \Delta)$ analyzed, whereas extreme CR $(0.05 \%$ glucose) showed deleterious effects on CLS (Figure 3a). Under the mild $\mathrm{CR}$ condition, $\mathrm{D}_{2} \mathrm{O}$ failed to further extend the prolonged lifespans of either wild-type or sir2 $\Delta$ mutants; yet, it still caused significant lifespan extension in tor $1 \Delta$ mutants (Figure 3a). Both SIR2 and TOR1 have reported roles in mediating CR responses in 
yeast: SIR2 mainly operates at mild CR by maintaining chromatin integrity in an NAD-dependent manner, whereas TOR1 works more efficiently under extreme $C R$ by modulating mitochondrial functions. ${ }^{4,8,9,23,25}$ Consistent with these findings, our observations suggest that $\mathrm{D}_{2} \mathrm{O}$ may mimic the mild $\mathrm{CR}$ condition to promote chronological longevity in yeast, possibly owing to their abilities to elicit catabolic activation. These findings together tether
$\mathrm{D}_{2} \mathrm{O}$-promoted longevity to calorie restriction and highlight the importance of delicate control of metabolism in yeast aging.

\section{CLS extension by $\mathrm{D}_{2} \mathrm{O}$ mediated by mitochondria}

In eukaryotes, mitochondria serve as the central hub to orchestrate material and energy metabolism, thus are the target a
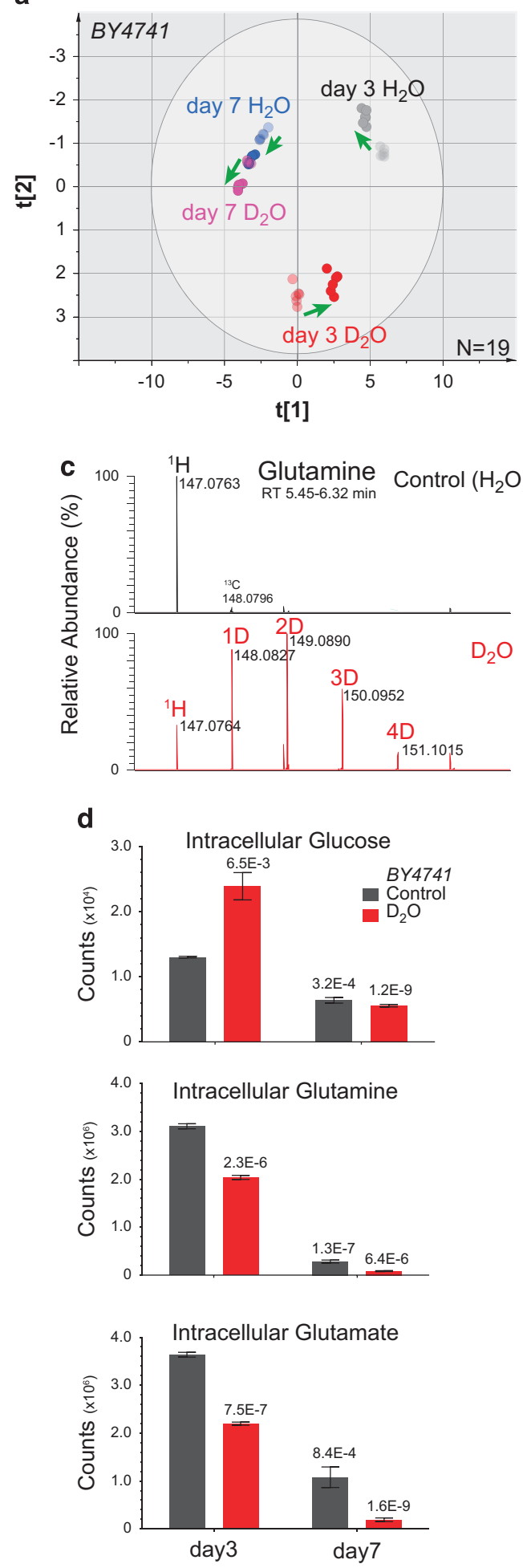

b
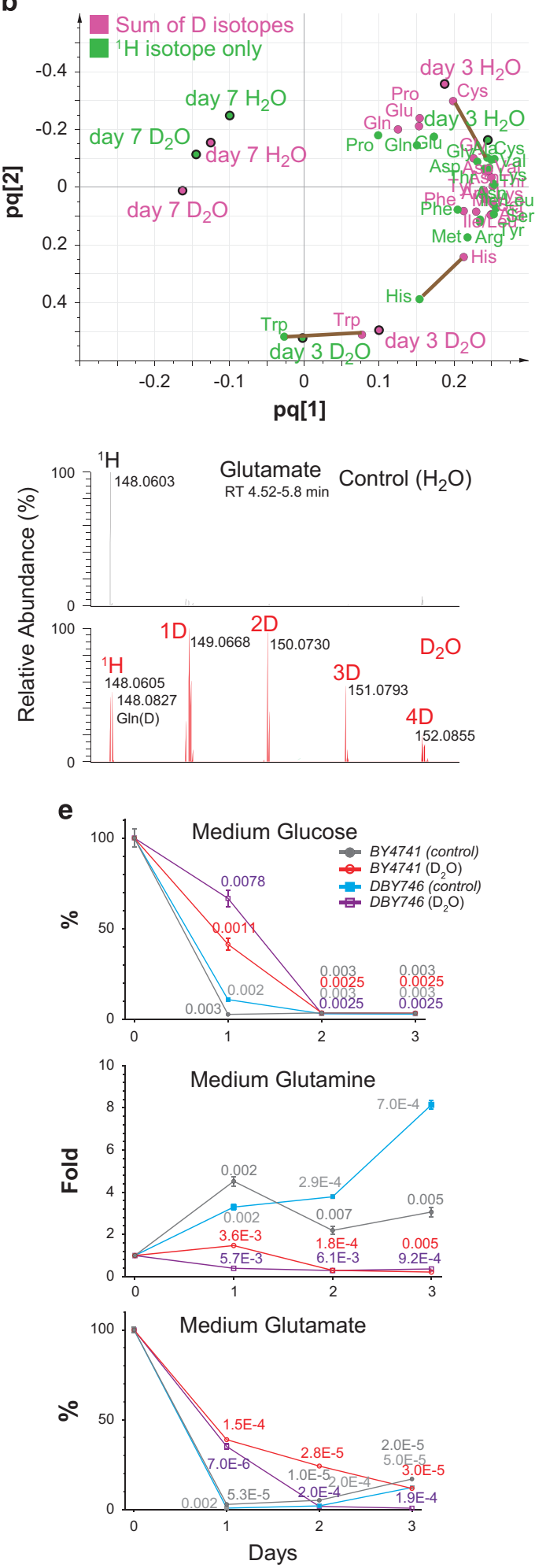
Figure 4. The metabolome shift by incorporation of deuterium through heavy water. See also Supplementary Figure S4. (a) The intracellular metabolome of BY4741 yeast cells upon $\mathrm{D}_{2} \mathrm{O}$ treatment at day 3 and day 7 was examined by LC-MS and clustered for all twenty proteinogenic amino acids (see Supplementary Figure S4A for global clustering). Two O2PLS-DA clustering models are superimposed to indicate the metabolome shift in amino-acid (AA) levels caused by $\mathrm{D}_{2} \mathrm{O}$ treatment. Green arrows indicate the direction from the model considering only ${ }^{1} \mathrm{H}$-isotopic AAs ( $\mathrm{H}$ isotope model, $\mathrm{R} 2 \mathrm{Y}=0.956, \mathrm{Q} 2=0.916$, half transparent) to the model that sums all deuterium isotopes for each $\mathrm{AA}$ (All D-isotope model, R2Y $=0.926, \mathrm{Q} 2=0.87$, opaque). Each circle represents one of two technical replicates each from three biological samples. (b) The contribution of individual AA to the O2PLS-DA clustering models in a. Each circle represents an AA. Brown lines linked three AAs that exhibited minimal deuterium incorporation. (c) Extensive deuterium incorporation in two metabolites glutamine and glutamate. Each panel shows one AA and its deuterated derivatives (red trace) in yeast cells (BY4741) grown in medium without $\mathrm{D}_{2} \mathrm{O}$ or with $50 \%$ 列 $\mathrm{O}$ at day 3. Accurate masses of relevant mass peaks $(\mathrm{m} / \mathrm{z})$ are indicated on each mass peak. (d) Intracellular metabolite levels shifted by $\mathrm{D}_{2} \mathrm{O}$ treatment. The intracellular sums of all hydrogen-isotopic metabolites for glucose, glutamine and glutamate from yeast cells (BY4741) at day 3 and day 7 , as summarized in $\mathbf{a}, \mathbf{b}$, are plotted $(n=3) . P$ values for $t$-test versus levels in day 3 control are indicated above each bar. Error bars = s.e.m. (e) Medium metabolite consumption pattern reversed by $\mathrm{D}_{2} \mathrm{O}$ treatment. The medium levels of glucose, glutamine and glutamate in the first 3 days of culture after inoculation for two strains (DBY746, BY4741, $n=3$ each). The $t$-test $P$ values are shown at each time point for comparison with starting medium levels. The control curves $\left(0 \% \mathrm{D}_{2} \mathrm{O}\right)$ for glutamine (middle panel) is the same as in Figure $1 \mathrm{~g}$. Error bars = s.e.m.

of many aging regulators. ${ }^{1,26}$ To test whether mitochondria mediate $\mathrm{D}_{2} \mathrm{O}$-promoted longevity, we conducted CLS assays on yeast strains with mitochondrial deficiency. The mip $1 \Delta$ strain carries deletion of the gene encoding the sole yeast mitochondrial DNA polymerase gamma subunit ${ }^{27}$ and are short-lived $(39.4 \%$ reduction in mean CLS versus wild type, $P<1 \mathrm{E}-10$; Figure $3 \mathrm{~b}$ ). Whereas $\mathrm{D}_{2} \mathrm{O}$ extended the mean CLS of wild-type (BY4741) significantly $(P=0.0022)$, it failed to increase the CLS of mip $1 \triangle$ mutants to the same magnitude (Figure $3 \mathrm{~b}$ ). We also generated petite $\left(r h o^{-}\right)$strains through ethidium bromide-induced mutagenesis. ${ }^{28,29}$ Petite strains are unable to grow on nonfermentable medium owing to loss of mitochondrial respiratory functions. Similarly, these petite strains did not show substantial lifespan extension in response to $\mathrm{D}_{2} \mathrm{O}$ treatment (Figure $3 \mathrm{~b}$ ). A modest but statistically significant CLS extension by $D_{2} \mathrm{O}$ in both mitochondrion-deficient conditions was observed ( $P$ values in Figure $3 \mathrm{~b}$ ), which could suggest anaerobic metabolism is also involved but is beyond the validity range of our CLS assays. Nevertheless, these results clearly demonstrate the strong dependence of the $\mathrm{D}_{2} \mathrm{O}$-promoted longevity on mitochondrial functions.

Heavy isotopes have been proposed to potentially confer cells chemical resistance to reactive oxygen species (ROS), ${ }^{14}$ a group of metabolic products that cause chemical damage and are implicated in aging. ${ }^{4,30,31}$ To test whether $D_{2} \mathrm{O}$ uptake affects endogenous ROS, we continuously monitored the total ROS and mitochondrial ROS generation in $\mathrm{D}_{2} \mathrm{O}$-treated live cells of three yeast strains using a fluorescence-based method (Supplementary Figure S3). Significant suppression of endogenous ROS generation by $\mathrm{D}_{2} \mathrm{O}$ was observed for both total and mitochondrial ROS, with maximal mitochondrial ROS suppression by $46 \%(P=2.7 \mathrm{E}-7)$ in DBY746 and maximal total ROS suppression by $62 \%(P=1.5 \mathrm{E}-4)$ in BY4743 (Figure 3c). These results strongly link $D_{2} O$ to ROS suppression. It is unknown whether $D_{2} \mathrm{O}$ suppresses $\mathrm{ROS}$ production by reducing free radical damages to intracellular targets, or by decreasing oxidative phosphorylation, a major source of intracellular ROS, or both. ${ }^{10,31,32}$ In summary, we found that $\mathrm{D}_{2} \mathrm{O}$ reduces chronological aging.

\section{Deuterium incorporation into the metabolome}

To investigate how $\mathrm{D}_{2} \mathrm{O}$ uptakes alters the metabolism, we analyzed the intracellular metabolome of yeast cells undergoing chronological aging upon $\mathrm{D}_{2} \mathrm{O}$ treatment by LC-MS. A total of 4008 metabolites formed discrete primary clusters between day 3 (start) and day 7 (50\% survival), which were also separated by $\mathrm{D}_{2} \mathrm{O}$ treatment along the secondary direction (Supplementary Figure S4a). Interestingly, 20 AAs also formed clusters showing a pattern very similar to that of the total metabolites (Figure 4a). To assess how deuterium incorporation may affect the metabolome, we compared two AA clustering models: one for those AAs that considers only the ${ }^{1} \mathrm{H}$-isotopic forms, and another using the sums of all D-isotopic forms for each AA (Figure 4a). Only minimal shift was observed whereas the overall cluster positioning remained almost unaffected (Figure 4a), suggesting that $\mathrm{D}_{2} \mathrm{O}$ did not skew the omic composition of AAs or the entire metabolome.

From the metabolomics perspective, AAs differed greatly in the extent of deuterium incorporation, with extensive incorporation in metabolically active AAs, such as Gln and Glu, but negligible incorporation in AAs that are non-limiting or auxotrophic, such as tryptophan and histidine (Figure $4 \mathrm{c}$ and Supplementary Figure $\mathrm{S} 4 \mathrm{~b}, \mathrm{c})$. Furthermore, extensive deuterium incorporation was not commensurate with metabolome clustering, which is evidenced by the close proximity of each AA between models, considering all ${ }^{1} \mathrm{H}$-isotopic forms and sum of all D-isotopic forms (Figure $4 \mathrm{~b}$ ). Only three AAs were observed with substantial shifts in distance, including tryptophan, histidine and cysteine, all of which had negligible deuterium incorporation (for example, see tryptophan in Supplementary Figure $S 4 b, c)$. These results implied that at least the other 17 AAs were connected by metabolism involving $\mathrm{D}_{2} \mathrm{O}$, which as a group showed no compositional response to $D_{2} \mathrm{O}$ uptake. The dynamics of metabolism may also explain the low toxicity of $\mathrm{D}_{2} \mathrm{O}$ in a range of organisms, including tolerance to pure $\mathrm{D}_{2} \mathrm{O}$ by yeast. ${ }^{33,34}$

To examine how $\mathrm{D}_{2} \mathrm{O}$ affects the metabolic capacity, we analyzed the intracellular and media levels of the same metabolites that are consumed by yeast or disposed of into the media. Surprisingly, the aging-associated decline in intracellular metabolites was not reversed by $\mathrm{D}_{2} \mathrm{O}$ (Figure $4 \mathrm{~b}$, d). Instead, $\mathrm{D}_{2} \mathrm{O}$ elicited an intracellular 'starvation' of AAs as early as day 3 (Figure 4b), which may mimic the pro-longevity effects by suppression of mTOR, a regulator of AA uptake. ${ }^{2,25}$ Intriguingly, the intracellular glucose was elevated by $\mathrm{D}_{2} \mathrm{O}$ only at early stage (Figure 4d). As the overall growth was not altered by $\mathrm{D}_{2} \mathrm{O}$ (Supplementary Figure S2b,e), the elevation in intracellular glucose at day 3 may result from improved metabolic conservation by $\mathrm{D}_{2} \mathrm{O}$, which could indicate a modest reduction in glycolysis or a more balanced coupling of glycolysis and its downstream aerobic respiration processes. This observation appears to corroborate the association of increased longevity with reduced insulin and IGF-1 signaling in mammals. ${ }^{1,35}$

$\mathrm{D}_{2} \mathrm{O}$ also alleviated nutrient consumption and metabolic waste disposal, which appears in favor of long-term survival under conditions with limited resources. In early growth stages before day $3, D_{2} \mathrm{O}$ uptake not only delayed the decline of medium nutrients, such as glucose and AAs (Glu, Asn, Asp and Thr); it also slowed the accumulation of certain metabolic waste, such as glutamine $^{16,17}$ and hypoxanthine (Figure $4 \mathrm{~d}$ and Supplementary Figure S4d), both are negatively associated with replicative lifespan. ${ }^{12,13}$ In contrast, the consumption of tryptophan and cysteine was unaffected by $\mathrm{D}_{2} \mathrm{O}$, coincident with the absence of 
deuterium incorporation in these metabolites (Figure 4b). These results indicate the metabolic basis for $\mathrm{D}_{2} \mathrm{O}$ uptake as a unique environmental intervention for long-term metabolic conservation and stabilization associated with longevity.

\section{DISCUSSION}

Stable heavy isotopes of common elements in living organisms are prevalent in nature. Although existing in much lower abundance than their light counterparts $\left(1.1 \% \mathrm{C}\right.$ for ${ }^{13} \mathrm{C}, 0.1 \% \mathrm{H}$ for ${ }^{2} \mathrm{H}$ or $\mathrm{D}$, and $0.5 \% \mathrm{~N}$ for ${ }^{15} \mathrm{~N}$ ), heavy isotopes produce remarkable kinetic difference because the gain in weight to charge increases the stability of heavy isotope-containing chemical bonds (e.g., by 5 - to 10 -fold for ${ }^{2} \mathrm{H}-\mathrm{C}$ over $\mathrm{H}-\mathrm{C}$ ), thus they may substantially slow the reactivity of the chemical bonds and make them less prone to chemical damages-this phenomenon is called kinetic isotope effects (KIE). ${ }^{14}$ The heavy isotopes may not affect biology over short periods because of their natural scarcity, but they could have strong effects after long-term accumulation resulting from massive flux, such as in the discrimination against ${ }^{13} \mathrm{C}$ in photosynthesis ${ }^{36}$ and in the metabolism of aging as described in this study (Figure 1). Owing to technical limitation, we only reported aging-associated heavy isotope decline in a group of small metabolites, AAs, but this decline may also exist in other metabolites. One noteworthy group are the lipids as we observed that many lipid species were completely deuterated upon $\mathrm{D}_{2} \mathrm{O}$ treatment (data not shown). Given the prevalent connections and robust dynamics of glutamine in energy and nitrogen metabolism, ${ }^{16,17}$ the heavy isotope decline in glutamine may represent the outcome of overall metabolic fluxes that are well conserved in all eukaryotes. ${ }^{37}$ The heavy isotope decline in yeast during aging may derive from preferential consumption that favors heavy isotopic metabolites or preferential disposal that favors light isotopic metabolites, or both, as suggested in the case of glutamine (Figure 1g). As such we speculate that catabolism may be more sensitive than anabolism in discriminating over isotopic metabolites in the case of $\mathrm{D}_{2} \mathrm{O}$, which might be related to the fact that water-splitting reactions (13\%) are twice as common as water-producing reactions $(7 \%)$ in yeast and even more in other organisms. ${ }^{19}$ Catabolic stimulation by $\mathrm{D}_{2} \mathrm{O}$ treatment may also explain its overlapping pro-longevity effects with calorie restriction as described in this study, as CR is thought to promote longevity through activation of catabolism. ${ }^{6}$ Altogether, our findings thus reported a natural decline in heavy isotopes in aging yeast, which may be used to assess similar metabolic decline associated with aging in other organisms including humans.

The dependence of $\mathrm{D}_{2} \mathrm{O}$ on mitochondria to extend lifespan highlights a pivotal role of metabolism in aging (Figure $3 \mathrm{~b}$ ). Despite the diversity of genetic adaptation in metabolism as a consequence of organismal evolution and environmental influence, calorie restriction appears to be the only anti-aging intervention that works across all experimental organisms, and perhaps in humans. ${ }^{1}$ By demonstrating that the $\mathrm{D}_{2} \mathrm{O}$-dependent lifespan extension overlaps with mild CR (Figure $3 a$ ) and requires mitochondria (Figure $3 \mathrm{~b}$ ), our results strongly support this notion that metabolic and mitochondrial regulators could regulate the process of aging in a number of organisms. ${ }^{1,38}$ Human population studies have linked higher resting metabolic rates to higher mortalities, ${ }^{39}$ suggesting that slowing the rate of basal metabolism might also retard human aging. It is still unclear how metabolism retardation, at the expense of possible delayed development and reduced survival, could benefit organismal lifespan. Heavy water generally retards growth and metabolism in many multicellular organisms, ${ }^{40}$ but it promoted chronological longevity in budding yeast (this study). Selective removal of mitochondria could also reduce growth in yeast. ${ }^{25,28,29}$ Heavy water has been shown to inhibit $\mathrm{H}_{2} \mathrm{O}_{2}$ production in rodent mitochondria. ${ }^{41}$ Higher levels of reactive oxygen species turnover, perhaps as the consequence of elevated mitochondrial activities, have recently been shown to negatively predict longevity of C. elegans. ${ }^{30}$ Our findings further confirmed that heavy water suppresses endogenous production of cellular and mitochondrial reactive oxygen species (Figure 3c). Altogether, these observations highlight a negative correlation of longevity with mitochondrial malfunctions, including metabolism deregulation, and mitochondria could serve as the major target for $\mathrm{D}_{2} \mathrm{O}$ to elicit prolongevity responses.

Although we do not fully understand the intracellular molecular mechanisms through which heavy isotopes retard aging, we speculate that the mode of actions may result directly from metabolic reactions, such as starvation-like responses and protection from ROS (Figures 3 and 4). We note that our method can only detect deuterium incorporation at the nonexchangeable element positions in each metabolite (Figures 1 and 4), and in the case of hydrogen, this includes $\mathrm{C}-\mathrm{H}$ bonds but not $\mathrm{N}-\mathrm{H}, \mathrm{O}-\mathrm{H}$ or $\mathrm{S}-\mathrm{H}$ bonds. Therefore, the observed stable incorporation of deuterium predominantly comes through metabolism and is less likely subject to potential contamination from experimental conditions of LC-MS. We showed that $\mathrm{D}_{2} \mathrm{O}$ does not alter the metabolome structure on a global basis with respect to aging (Figure 4), nor does it require known genetic regulators of CR or DR to extend yeast lifespan (Figure 2), suggesting in each case that the overall biological functions are not extensively altered at the genetic levels. Conversely, the dependence of CLS extension by $\mathrm{D}_{2} \mathrm{O}$ on $\mathrm{CR}$ and mitochondrial functions (Figure 3 ) and the failure of CLS extension through ${ }^{2} \mathrm{H}$-glucose feeding (representing lesser $\mathrm{KIE})$ (Figure 2) strongly argue that metabolic actions are critical to longevity promotion by heavy isotopes. Besides, $\mathrm{D}_{2} \mathrm{O}$ slows the pace for both nutrient consumption and waste disposal (Figure 4 and Supplementary Figure S4) but not the growth (Supplementary Figure S2), suggesting that heavy isotopes act bilaterally on the metabolism in favor of metabolic conservation, which may ultimately lead to better long-term sustainability and longevity. Although we only showed evidence for hydrogen isotopes, other elements may also prove to be useful when an effective strategy for delivery is devised. It is also possible that the administration of heavy isotopes might extend lifespan in higher organisms, although it remains unknown whether they might interfere with developmental and biological functions in such organisms. However, it has been shown that the $\mathrm{D}_{2} \mathrm{O}$ doses that extend lifespan do not affect the growth of yeast (Figure 2) or the fertility of fruit flies. ${ }^{42}$

In conclusion, the decline of heavy isotope content in metabolites represents a novel feature of aging and could be a new target to promote longevity by environmental intervention. Specifically, supplementation of heavy isotopes through metabolism could provide a new avenue to control aging and age-associated diseases.

\section{MATERIALS AND METHODS}

\section{Strains}

Strains are listed in Supplementary Table S1. Gene deletion strains were retrieved from the Yeast Genome Deletion Project collection, and all strains were generated in the BY4741 background.

\section{Media}

Yeast SDC media were prepared as previously described ${ }^{9,43}$ with fortified fourfold AAs in surplus, which is auxotrophic to the yeast strains used. YPAD media contained yeast extract $(1 \% \mathrm{w} / \mathrm{v})$, peptone $(2 \%, \mathrm{w} / \mathrm{v})$, dextrose $(2 \% \mathrm{w} / \mathrm{v})$, and adenine hemisulfate $(60 \mathrm{mg} / \mathrm{l})$. Heavy water (deuterium oxide, 99.9 atom \% D) was purchased from Sigma-Aldrich (St Louis, MO, USA), and was filtered with $0.2-\mu \mathrm{m}$ PES filter before use. $D_{7}$-glucose (DGlucose-1,2,3,4,5,6,6-D $\mathrm{D}_{7}$ ) was purchased from Sigma-Aldrich (Cat. 552003), and prepared in $40 \% \mathrm{w} / \mathrm{v}$ stock solution before use. 
Metabolomics sample preparation

Yeast culture were centrifuged at $5,000 \mathrm{~g}$ for $5 \mathrm{~min}$ at room temperature to collect cells, followed by twice wash in water (MS grade). The cell pellet was flash frozen in dry ice bath until processing. For LC/MS analysis, frozen cell pellet (or medium) of equal cell counts (by absorption at $600 \mathrm{~nm}$ ) was mixed with dry ice-cool $80 \%$ methanol (mass-spec grade) at the same ratio for the same sample batches (usually 1 vol: 10 vol solvent), and then quickly thawed on heat block set at $50^{\circ} \mathrm{C}$ for $5 \mathrm{~min}$. The suspension was then processed by three rounds of 1-min vortex at max speed, chilled briefly on ice. The mixture was incubated at $4^{\circ} \mathrm{C}$ for $15 \mathrm{~min}$ before centrifugation at $20,000 \mathrm{~g}$ for $20 \mathrm{~min}$ at $4{ }^{\circ} \mathrm{C}$. The supernatant was stored at $-20^{\circ} \mathrm{C}$ and used as metabolite extract for LC-MS analysis. For LC-MS analysis, the metabolite extract was transferred to $150-\mu$ l deactivated glass insert housed in Waters 2-ml brown MS vials. Chemical standard solution was prepared from $1 \times$ synthetic complete mixture from Sigma-Aldrich (Y1501).

\section{LC/MS acquisition}

LC/MS analysis was performed in a platform that consists of Waters UPLC-coupled Exactive Orbitrap Mass Spectrometer (Thermo, Waltham, MA, USA), using a mix-mode OPD2 HP-4B column $(4.6 \times 50 \mathrm{~mm})$ with a $4.6 \times 10 \mathrm{~mm}$ guard column (Shodex, Showa Denko, Tokyo, Japan). The column temperature was maintained at $45^{\circ} \mathrm{C}$. The sample chamber was maintained at $4{ }^{\circ} \mathrm{C}$. The binary mobile phase solvents were: $\mathrm{A}, 10 \mathrm{mM}$ $\mathrm{NH}_{4} \mathrm{OAc}$ in 10:90 Acetonitrile:water; $\mathrm{B}, 10 \mathrm{mM} \mathrm{NH}_{4} \mathrm{OAc}$ in 90:10 Acetonitrile: water. Both solvents were modified with $10 \mathrm{mM}$ HOAc $(\mathrm{pH}$ 4.75) for positive mode acquisition, or $10 \mathrm{mM} \mathrm{NH}_{4} \mathrm{OH}(\mathrm{pH} 7.25)$ for negative mode. The flow was set as: flow rate, $0.1 \mathrm{ml} / \mathrm{min} ; 0-15 \mathrm{~min}, 99 \% \mathrm{~A}, 15-18 \mathrm{~min}$, $99 \%$ to $1 \% \mathrm{~A} ; 18-24 \mathrm{~min}, 1 \% \mathrm{~A} ; 24-25 \mathrm{~min}, 1 \%$ to $99 \% \mathrm{~A} ; 25-30 \mathrm{~min}, 99 \% \mathrm{~A}$. The MS acquisition was in profile mode and performed with an ESI probe, operating with capillary temperature at $275^{\circ} \mathrm{C}$, sheath gas at 40 units, spray voltage at $3.5 \mathrm{kV}$ for positive mode and $3.1 \mathrm{kV}$ for negative mode, Capillary voltage at $30 \mathrm{~V}$, tube lens voltage at $120 \mathrm{~V}$ and Skimmer voltage at $20 \mathrm{~V}$. The mass scanning used 100,000 mass resolution, high dynamic range for AGC Target, $500 \mathrm{~ms}$ as Maximum Inject Time and 70-1,000 m/z as the scan range.

\section{LC/MS data analysis}

The raw LC/MS data files were centroided with PAVA program ${ }^{44}$ and converted to mzXML format by an in-house $\mathrm{R}$ script (distribution upon request). Mass feature extraction was performed with XCMS v1.30.3. ${ }^{45}$ The mass features were then manually searched against the Metlin metabolite database using 5 p.p.m. mass accuracy. Retention time matching with compounds in the standard mixture was also performed for a portion of the metabolite hits. The scored mass features were clustered with SIMCA v13.03 (Umetric, Malmö, Sweden).

\section{Chronological lifespan assay}

The CLS assay was carried out as previously described. ${ }^{9,43}$ Fresh colonies were inoculated into SDC media. Overnight culture was seeded into media containing $\mathrm{H}_{2} \mathrm{O}$ or respective doses of $\mathrm{D}_{2} \mathrm{O}$, and the cultures were kept on a roller drum at $28^{\circ} \mathrm{C}$. Starting from day 3 and then every other day thereafter, a small amount of the culture was taken out and diluted and plated on YPD plates to determine colony formation after 2 days of incubation at $30^{\circ} \mathrm{C}$. Day 3 is considered as the point of $100 \%$ survival. Kaplan-Meier Estimator was used to calculate the mean lifespan, using the online software OASIS, ${ }^{46}$ the $P$ values were calculated by Log-Rank Test.

\section{Metabolic pathway enrichment analysis}

The KEGG IDs of those metabolites with high discriminatory power were used for pathway enrichment analysis with IMPaLA. ${ }^{47}$ Manual measurement of monoisotopic metabolites was performed in Thermo Xcalibur v2.1 software, with the capability to simulate monoisotopic peaks containing heavy isotopes.

\section{Petite clone generation}

The petite strains were generated following established protocol. ${ }^{28,29}$ Fresh overnight cultures were diluted 1:10 with YPD medium, and treated with ethidium bromide $(10 \mu \mathrm{g} / \mathrm{ml})$ for $3 \mathrm{~h}$ at $28^{\circ} \mathrm{C}$. The culture was then plated on YP plates containing $0.1 \%$ glucose and $3 \%$ glycerol as carbon source for petite and grande selection. The petite phenotype was confirmed by the failure of treated cells to grow on YP media containing only $3 \%$ glycerol.

Plate-based growth assay

Yeast growth assay was performed in 96-well plate format. Fresh overnight cultures were washed twice in water and inoculated in respective experimental medium. The plate was then incubated in a Tecan plate reader at $30^{\circ} \mathrm{C}$ with agitation. Optical density at $600 \mathrm{~nm}$ was determined every 15 min during a period of 2 to 3 days. The growth curve parameters were deduced by $\mathrm{R}$ package grofit. ${ }^{48}$

Plate-based assay for reactive oxygen species production in vivo Yeast cell cultures at stationary phase were added to 96-well plate. Two fluorescent dyes, CellROX deep red (total ROS, C10422, Life Technologies, Carlsbad, CA, USA) and MitoSOX (mitochondrial ROS, M36008, Life Technologies), were added separately to final concentrations of $5 \mu \mathrm{M}$. Hoechst 33342 (H3570, Life Technologies) was added to $5 \mu \mathrm{g} / \mathrm{ml}$ to monitor the cell density. The cultures were incubated at $30^{\circ} \mathrm{C}$ with shaking on a Tecan plate reader. The fluorescence was monitored every $15 \mathrm{~min}$ for $25 \mathrm{~h}$ with fluorescence settings: CelROX, Ex/Em 640/665 nm; MitoSOX, Ex/ Em: 510/580 nm; Hoechst 33342, Ex/Em: 350/461 nm. Band width was $10 \mathrm{~nm}$. Fresh media with respective fluorescence dyes were used as blank controls for autofluorescence.

\section{Cell volume measurement}

Fresh overnight yeast culture was added at a ratio of 1:400 to SDC medium containing different amounts of $\mathrm{D}_{2} \mathrm{O}$. On day 2 , the mean cell volumes were determined with a Coulter counter after a 2,000-fold dilution.

\section{ACKNOWLEDGEMENTS}

This work was supported by NIH grant (5R01GM06248012) and California Institute for Regenerative Medicine grant (RB4-06087) to M.P.S. The authors would like to thank Drs Valter Longo and Min Wei for helps in performing yeast CLS assays; Drs Peichuan Zhang, Anne Brunet, and Ms Shuo Han for stimulating discussion; Drs Gavin Sherlock and Katja Schwartz for help in cell volume measurement; Drs Peichuan Zhang, Xin Wang, Wei Jiang, Haiyang Li, and Xianjun Zhu for critical reading and comments during manuscript preparation.

\section{CONTRIBUTIONS}

X.L. designed and carried out the experiments and performed data analysis. X.L. and M.P.S. wrote the manuscript. M.P.S. supervised and helped to design the research.

\section{COMPETING INTERESTS}

The authors declare no conflict of interest.

\section{REFERENCES}

1. Lopez-Otin, C., Blasco, M. A., Partridge, L., Serrano, M. \& Kroemer, G. The hallmarks of aging. Cell 153, 1194-1217 (2013).

2. Powers, R. W. 3rd, Kaeberlein, M., Caldwell, S. D., Kennedy, B. K. \& Fields, S. Extension of chronological life span in yeast by decreased TOR pathway signaling. Genes Dev. 20, 174-184 (2006).

3. Kahn, B. B., Alquier, T., Carling, D. \& Hardie, D. G. AMP-activated protein kinase: ancient energy gauge provides clues to modern understanding of metabolism. Cell Metab. 1, 15-25 (2005).

4. Lin, S. J., Defossez, P. A. \& Guarente, L. Requirement of NAD and SIR2 for life-span extension by calorie restriction in Saccharomyces cerevisiae. Science $\mathbf{2 8 9}$ 2126-2128 (2000).

5. Jazwinski, S. M. Yeast longevity and aging--the mitochondrial connection. Mech. Ageing Dev. 126, 243-248 (2005).

6. Jiang, J. C., Jaruga, E., Repnevskaya, M. V. \& Jazwinski, S. M. An intervention resembling caloric restriction prolongs life span and retards aging in yeast. FASEB J. 14, 2135-2137 (2000).

7. Kaeberlein, M. Lessons on longevity from budding yeast. Nature 464, 513-519 (2010).

8. Kaeberlein, M., Kirkland, K. T., Fields, S. \& Kennedy, B. K. Sir2-independent life span extension by calorie restriction in yeast. PLoS Biol. 2, E296 (2004).

9. Fabrizio, P. et al. Sir2 blocks extreme life-span extension. Cell 123, 655-667 (2005). 
10. Lesnefsky, E. J. \& Hoppel, C. L. Oxidative phosphorylation and aging. Ageing Res. Rev. 5, 402-433 (2006)

11. Nakagawa, T., Lomb, D. J., Haigis, M. C. \& Guarente, L. SIRT5 Deacetylates carbamoyl phosphate synthetase 1 and regulates the urea cycle. Cell 137, 560-570 (2009).

12. Kamei, Y., Tamada, Y., Nakayama, Y., Fukusaki, E. \& Mukai, Y. Changes in transcription and metabolism during the early stage of replicative cellular senescence in budding yeast. J. Biol. Chem. 289, 32081-32093 (2014).

13. Yoshida, R. et al. Metabolomics-based systematic prediction of yeast lifespan and its application for semi-rational screening of ageing-related mutants. Aging Cell $\mathbf{9}$, 616-625 (2010).

14. Shchepinov, M. S. Reactive oxygen species, isotope effect, essential nutrients, and enhanced longevity. Rejuvenation Res. 10, 47-59 (2007).

15. Ljungdahl, P. O. \& Daignan-Fornier, B. Regulation of amino acid, nucleotide, and phosphate metabolism in Saccharomyces cerevisiae. Genetics 190, 885-929 (2012).

16. Boer, V. M., Crutchfield, C. A., Bradley, P. H., Botstein, D. \& Rabinowitz, J. D. Growth-limiting intracellular metabolites in yeast growing under diverse nutrient limitations. Mol. Biol. Cell 21, 198-211 (2010).

17. Yuan, J., Fowler, W. U., Kimball, E., Lu, W. \& Rabinowitz, J. D. Kinetic flux profiling of nitrogen assimilation in Escherichia coli. Nat. Chem. Biol. 2, 529-530 (2006).

18. Ong, S. E. \& Mann, M. A practical recipe for stable isotope labeling by amino acids in cell culture (SILAC). Nat. Protoc. 1, 2650-2660 (2006).

19. Caspi, R. et al. The MetaCyc database of metabolic pathways and enzymes and the BioCyc collection of Pathway/Genome Databases. Nucleic Acids Res. 42, D459-D471 (2014)

20. Taylor, G. W. \& Harvey, E. N. Respiration of yeast in water containing deuterium oxide. Exp. Biol. Med. 31, 954-957 (1934).

21. Gomes, P., Sampaio-Marques, B., Ludovico, P., Rodrigues, F. \& Leao, C. Low auxotrophy-complementing amino acid concentrations reduce yeast chronological life span. Mech. Ageing Dev. 128, 383-391 (2007).

22. Kaeberlein, M., Kirkland, K. T., Fields, S. \& Kennedy, B. K. Genes determining yeast replicative life span in a long-lived genetic background. Mech. Ageing Dev. 126, 491-504 (2005).

23. Anderson, R. M. et al. Yeast life-span extension by calorie restriction is independent of NAD fluctuation. Science 302, 2124-2126 (2003).

24. Kaeberlein, M. et al. Regulation of yeast replicative life span by TOR and Sch9 in response to nutrients. Science 310, 1193-1196 (2005).

25. Bonawitz, N. D., Chatenay-Lapointe, M., Pan, Y. \& Shadel, G. S. Reduced TOR signaling extends chronological life span via increased respiration and upregulation of mitochondrial gene expression. Cell Metab. 5, 265-277 (2007).

26. Breitenbach, M. et al. The role of mitochondria in the aging processes of yeast. Subcell. Biochem. 57, 55-78 (2012).

27. Genga, A., Bianchi, L. \& Foury, F. A nuclear mutant of Saccharomyces cerevisiae deficient in mitochondrial DNA replication and polymerase activity. J. Biol. Chem. 261, 9328-9332 (1986).

28. Goldring, E. S., Grossman, L. I., Krupnick, D., Cryer, D. R. \& Marmur, J. The petite mutation in yeast. Loss of mitochondrial deoxyribonucleic acid during induction of petites with ethidium bromide. J. mol. biol. 52, 323-335 (1970).

29. Slonimski, P. P., Perrodin, G. \& Croft, J. H. Ethidium bromide induced mutation of yeast mitochondria: complete transformation of cells into respiratory deficient non-chromosomal "petites". Biochem. Biophys. Res. Commun. 30, 232-239 (1968).
30. Shen, E. Z. et al. Mitoflash frequency in early adulthood predicts lifespan in Caenorhabditis elegans. Nature 508, 128-132 (2014).

31. Liochev, S. I. Reactive oxygen species and the free radical theory of aging. Free Radic. Biol. Med. 60, 1-4 (2013).

32. Kirkinezos, I. G. \& Moraes, C. T. Reactive oxygen species and mitochondrial diseases. Semin. Cell Dev. Biol. 12, 449-457 (2001).

33. Lewis, G. N. The Biology of Heavy Water. Science 79, 151-153 (1934).

34. Taylor, G. W. \& Harvey, E. N. Respiration of yeast in water containing deuterium oxide. Proc. Soc. Exp. Biol. Med. 31, 954-957 (1934).

35. Parrella, E. \& Longo, V. D. Insulin/IGF-I and related signaling pathways regulate aging in nondividing cells: from yeast to the mammalian brain. Sci. World J. 10, 161-177 (2010).

36. Farquhar, G. D., Ehleringer, J. R. \& Hubick, K. T. Carbon isotope discrimination and photosynthesis. Annu. Rev. Plant Phys. 40, 503-537 (1989).

37. Peregrin-Alvarez, J. M., Sanford, C. \& Parkinson, J. The conservation and evolutionary modularity of metabolism. Genome Biol. 10, R63 (2009).

38. Kenyon, C. J. The genetics of ageing. Nature 464, 504-512 (2010).

39. Jumpertz, R. et al. Higher energy expenditure in humans predicts natural mortality. J. Clin. Endocrinol. Metab. 96, E972-E976 (2011).

40. Fox, D. L. Heavy water and metabolism. Quart. Rev. Biol. 9, 342-346 (1934).

41. Pomytkin, I. A. \& Kolesova, O. E. Relationship between natural concentration of heavy water isotopologs and rate of $\mathrm{H} 2 \mathrm{O} 2$ generation by mitochondria. Bull. Exp. Biol. Med. 142, 570-572 (2006).

42. Hammel, S. C., East, K., Shaka, A. J., Rose, M. R. \& Shahrestani, P. Brief early-life non-specific incorporation of deuterium extends mean life span in Drosophila melanogaster without affecting fecundity. Rejuvenation Res. 16, 98-104 (2013).

43. Wei, M., Madia, F. \& Longo, V. D. Studying age-dependent genomic instability using the $S$. cerevisiae chronological lifespan model. J. Vis. Exp. 55, e3030-e3036 (2011)

44. Guan, S., Price, J. C., Prusiner, S. B., Ghaemmaghami, S. \& Burlingame, A. L. A data processing pipeline for mammalian proteome dynamics studies using stable isotope metabolic labeling. Mol. Cell Proteom. 10(M111): 010728 (2011).

45. Smith, C. A., Want, E. J., O'Maille, G., Abagyan, R. \& Siuzdak, G. XCMS: processing mass spectrometry data for metabolite profiling using nonlinear peak alignment, matching, and identification. Anal. Chem. 78, 779-787 (2006).

46. Yang, J. S. et al. OASIS: online application for the survival analysis of lifespan assays performed in aging research. PLoS ONE 6, e23525 (2011).

47. Kamburov, A., Cavill, R., Ebbels, T. M., Herwig, R. \& Keun, H. C. Integrated pathway-level analysis of transcriptomics and metabolomics data with IMPaLA. Bioinformatics 27, 2917-2918 (2011).

48. Kahm, M., Hasenbrink, G., Lichtenberg-Fraté, H., Ludwig, J. \& Kschischo, M. grofit: Fitting Biological Growth Curves with R. J. Stat. Softw. 33, 1-21 (2010).

\section{c) (i)}

This work is licensed under a Creative Commons Attribution 4.0 International License. The images or other third party material in this article are included in the article's Creative Commons license, unless indicated otherwise in the credit line; if the material is not included under the Creative Commons license, users will need to obtain permission from the license holder to reproduce the material. To view a copy of this license, visit http://creativecommons.org/licenses/ by/4.0/

Supplemental Information accompanies the paper on the npj Aging and Mechanisms of Disease website (http://www.nature.com/npjamd) 